\title{
Dependence of Acute Myeloid Leukemia on Adhesion within the Bone Marrow Microenvironment
}

\author{
Pamela S. Becker \\ Division of Hematology, Institute for Stem Cell and Regenerative Medicine, University of Washington, Campus Box 358056, \\ 815 Mercer Street N415, Seattle, WA 98109, USA \\ Correspondence should be addressed to Pamela S. Becker, pbecker@u.washington.edu
}

Received 27 September 2011; Accepted 20 October 2011

Academic Editors: T. Darribere and B. Savani

Copyright ( 2012 Pamela S. Becker. This is an open access article distributed under the Creative Commons Attribution License, which permits unrestricted use, distribution, and reproduction in any medium, provided the original work is properly cited.

Acute myeloid leukemia (AML) cells home to the endosteal region of the bone marrow. They interact with bone marrow stromal components including extracellular matrix proteins, glycosaminoglycans, and stromal cells, by which they derive proliferative and growth inhibitory signals. Furthermore, adhesion to marrow stroma confers chemotherapy drug resistance and thereby promotes leukemia survival. A subpopulation of the leukemic blasts, known as leukemia stem cells, that are capable of propagating the leukemia, remain sheltered in the bone marrow microenvironment, exhibit resistance to chemotherapy, and serve as the origin of relapse after a variable period of remission. Detachment of these cells from the bone marrow in combination with chemotherapy may improve the outcome of therapy for AML.

\section{Adhesion and Leukemia Biology}

Adhesive properties of leukemia cells are likely responsible for the complication of leukostasis in AML as well as leukemic meningitis, leukemia cutis, extramedullary leukemia, and formation of chloromas. Three receptors, VLA- (very late antigen-) 4, CXCR4, and CD44, play a critical role in normal stem cell homing and also appear to be paramount to the homing of AML cells to, or retention within, the bone marrow. VLA4 is the $\alpha_{4} \beta_{1}$ integrin that mediates adhesion to alternatively spliced fibronectin and cellular vascular cell adhesion molecule-1 (VCAM1). CXCR4 is a chemokine receptor for stromal derived factor-1 (SDF-1) also known as CXCL12. CD44 is a hyaluronic acid receptor that is an E selectin ligand expressed by hematopoietic stem cells known as HCELL when properly glycosylated [1]. The VLA4/VCAM-1 pathway has been implicated in the attachment of leukemic blasts to the vessel wall [2]. Both CXCR-4 and VLA-4 mediate migration of AML blasts $[3,4]$. The role of CXCR4 in leukemia retention was illustrated by experiments that demonstrated reduction of primary human AML cell numbers previously engrafted in immunodeficient NODscid mice with antibody to CXCR-4 [5]. In contrast, there was no decrease in normal human CD34+ cell numbers in mice engrafted with normal human cord blood mononuclear cells after treatment with the anti-CXCR4 antibody [5]. This finding highlights the exquisite ongoing dependence of the engraftment of human AML in immunodeficient mice on CXCR-4. In addition, the CD44 hyaluronic acid receptor is involved in homing of normal human CD34+ cells [6]. Similarly, administration of an antibody to CD44 blocked engraftment of AML cells in NOD-scid mice [7]. Furthermore, high level expression of CD44 by leukemia cells was sufficient to generate leukemia by leukemia-initiating cells even after withdrawal of overexpression of the HoxA10 gene that initiated the leukemia [8]. Thus, at least three adhesion mechanisms, CXCR4/SDF1 (CXCL12), VLA-4/VCAM-1 or fibronectin, and CD44/ligand, function in acute myeloid leukemia migration, retention, and survival (Figure 1).

Not only are leukemia cells dependent on the bone marrow stroma for survival, but also they are capable of distorting normal bone marrow niches in a manner that affects normal hematopoietic progenitor cells [9]. The endo-steal region was the location identified as the site of homing of chemotherapy-resistant AML stem cells [10], supporting the concept of localizing niches for certain cell types, and this location is the same region as a niche for normal homing hematopoietic stem cells [11]. 




Figure 1: Leukemia cell adhesion and chemokine receptors in stromal interactions. CXCR4, VLA-4, and CD44 play critical roles in leukemia cell homing and migration.

\section{Adhesion and Chemotherapy Resistance}

Adhesion of acute myeloid leukemia cells confers resistance to several chemotherapy agents, including cytarabine, one of the most active agents in AML. This ability is known as environment-mediated drug resistance (EMDR) [12]. The theory is that this capacity, particularly when possessed by leukemia stem cells, for example, gives rise to minimal residual disease, which in turn, is the origin of relapse after a period of genetic instability and acquisition of more complex drug resistance. Growth of AML cells on HS-5 stroma reduced daunorubicin- or cytarabine-induced apoptosis [13]. Adhesion of U937 to fibronectin via $\beta_{1}$ integrins inhibits mitoxantrone- and etoposide-induced apoptosis [14]; similarly, adhesion of U937 or HL60 leukemia cell lines to fibronectin inhibited daunorubicin or cytarabine induced apoptosis [15]. Adhesion of primary patient AML cells to fibronectin or immobilized VCAM-1 conferred resistance to cytarabine or daunorubcin plus cytarabine [16]. Agents that block adhesion mediated by VLA-4, including a fibronectin peptide [17], antibody to VLA-4 [15, 16], soluble VCAM1 [16], a small molecule inhibitor of VLA-4 [18], or a peptide inhibitor of CXCR-4 [19] all overcame adhesion mediated chemotherapy resistance. Moreover, a peptide inhibitor of the chemokine receptor, CXCR4, exhibited direct cytotoxicity against AML and multiple myeloma cells in vitro and in xenografts [20]. The CXCR4 inhibitor AMD3100 worked synergistically with histone deacetylase inhibitor panobinostat to induce apoptosis of AML cells in vitro [21]. Another CXCR4 inhibitor, AMD3465, interfered with chemotaxis of AML cells toward SDF1 in vitro, prevented SDF1-induced activation of survival pathways in AML cells, caused mobilization of human leukemia cells in immunodeficient mouse xenografts, and enhanced the activity of sorafenib in Flt3-positive AML [22]. In an in vivo murine model of acute promyelocytic leukemia, AMD3100 mobilized leukemia cells into the blood and, in combination with cytarabine, reduced leukemic burden and prolonged animal survival [23]. These latter two studies demonstrated the proof of principle that the concept that a combination of a CXCR4 inhibitor with chemotherapy or targeted therapy was efficacious in enhancing leukemia cytotoxicity in vivo.

Several potential mechanisms have been proposed for the ability of integrin-mediated signaling to protect from chemotherapy toxicity that involve activation of survival pathways or inhibition of apoptosis. The specific pathways include activation of the PI3 K/Akt/bcl-2 pathway [15], an interaction between Wnt and adhesion-dependent signaling pathways [24], and increased degradation of proapoptotic bcl-2 family member Bim [25]. Integrin-linked kinase (ILK) also plays a role in the activation of Akt upon adhesion of AML cells $[26,27]$.

\section{Chemokine Receptor or Adhesion Receptor Expression and Prognosis}

Expression of each of the two receptors, VLA-4 or CXCR4, has been associated with prognosis in AML; the former is correlated with better survival, and the latter portends worse survival. Several studies showed that high-level expression of CXCR4 was associated with poor prognosis in AML. As described earlier, CXCR-4 was demonstrated to have a pivotal role in the homing, migration, and development of human AML in the NODscid murine mutant [5]. Although not all AML patient cells tested exhibited surface expression of CXCR-4 with average expression 24\%, all AML cells analyzed uniformly exhibited internal expression of CXCR- 4 after permeabilization and labeling [5]. AML patients with high-level $(\geq 20 \%)$ expression of CXCR-4 by the CD34+ population exhibited reduced overall survival and relapse free survival [28]. By multivariate Cox regression analysis, high CXCR-4 expression had a relative risk for relapse of $13.4(P<0.001)$ [28]. Furthermore, an independent study also corroborated that high-level CXCR-4 expression predicted overall and event-free survival in patients with normal karyotype and unmutated Flt3 status [29], and lower expression of CXCR4 correlated with longer relapse-free and overall survival [30] or higher complete remission rate [31]. Presence of functional circulating CXCR4 bearing microparticles was correlated with high white blood count in AML patients and was proposed to be involved in AML progression, possibly by promoting dissemination of leukemia [32].

In contrast to CXCR4 expression, high-level VLA-4 expression has the opposite effect on prognosis in AML. Higher functional expression of VLA-4 was shown to correlate with longer survival for newly diagnosed adult AML [16]. Furthermore, higher expression of VLA- 4 by flow cytometry correlated with better prognosis of pediatric AML patients [33]. These two large studies are in contrast with an earlier, smaller trial that suggested that VLA-4 expression conferred poor prognosis [15]. The precise mechanism for this improved survival is unknown, but one hypothesis is that as soluble VCAM-1 (sVCAM-1) levels are elevated in AML [34], the AML blasts may be dislodged from the bone marrow due to binding of sVCAM-1 and thus be more susceptible to chemotherapy.

\section{Clinical Trials of Adhesion Inhibitors in AML}

There are several ongoing clinical trials utilizing this novel concept of combining agents that mobilize leukemia with chemotherapy (Table 1). For example, there is an ongoing multicenter phase I trial of plerixafor in combination with 
TABLE 1: Clinical trials of combinations of adhesion inhibitors and chemotherapy for AML.

\begin{tabular}{|c|c|c|}
\hline Title & Clinicaltrials.gov designation & Institution or sponsor \\
\hline $\begin{array}{l}\text { Study of plerixafor combined with } \\
\text { cytarabine and daunorubicin in patients } \\
\text { with newly diagnosed acute myeloid } \\
\text { leukemia }\end{array}$ & NCT00990054 & Multicenter-Genzyme-Sanofi \\
\hline $\begin{array}{l}\text { First in human study to determine the } \\
\text { safety, tolerability, and preliminary } \\
\text { effectiveness of MDX-1338 (BMS936564) } \\
\text { in subjects with acute myelogenous } \\
\text { leukemia (AML) }\end{array}$ & NCT01120457 & Multicenter-Bristol-Myers Squibb \\
\hline $\begin{array}{l}\text { Granulocyte colony-stimulating factor } \\
\text { (G-CSF) and plerixafor plus sorafenib for } \\
\text { acute myelogenous leukemia (AML) with } \\
\text { FLT3 mutations }\end{array}$ & NCT00943943 & MD Anderson Cancer Center \\
\hline $\begin{array}{l}\text { Chemosensitization with plerixafor plus } \\
\text { G-CSF in acute myeloid leukemia }\end{array}$ & NCT00906945 & Washington University \\
\hline $\begin{array}{l}\text { IV plerixafor with mitoxantrone } \\
\text { etoposide and cytarabine for acute } \\
\text { myeloid leukemia (AML) }\end{array}$ & NCT01027923 & Washington University \\
\hline $\begin{array}{l}\text { Plerixafor and clofarabine in frontline } \\
\text { treatment of elderly patients with acute } \\
\text { myelogenous leukemia (AML) }\end{array}$ & NCT01160354 & MD Anderson Cancer Center \\
\hline
\end{tabular}

standard induction " $7+3$ " chemotherapy in AML including high-dose daunorubicin $90 \mathrm{mg} / \mathrm{m}^{2}$ daily for three days. There is also an ongoing multicenter phase I trial of an anti-CXCR-4 antibody in combination with mitoxantrone, etoposide, and cytarabine for relapsed/refractory AML. As these inhibitors enter the clinic, we will ascertain their ability to mobilize AML out of the protected marrow microenvironment and determine if this approach improves outcome of patients with new diagnosis or relapsed/refractory AML.

\section{Future Prospects}

In summary, there may be several critical mechanisms for adhesion of AML within the bone marrow, and discovery of novel mechanisms and novel inhibitors targeting disruption of adhesion may provide a significant advance in the treatment of AML.

\section{Acknowledgment}

Dr. P. S. Becker is supported in part by a research grant from the Translational Research Program of the Leukemia and Lymphoma Society.

\section{References}

[1] J. S. Merzaban, M. M. Burdick, S. Z. Gadhoum et al., "Analysis of glycoprotein E-selectin ligANDs on human and mouse marrow cells enriched for hematopoietic stem/progenitor cells," Blood, vol. 118, no. 7, pp. 1774-1783, 2011.

[2] A. Stucki, A. S. Rivier, M. Gikic, N. Monai, M. Schapira, and O. Spertini, "Endothelial cell activation by myeloblasts: molecular mechanisms of leukostasis and leukemic cell dissemination," Blood, vol. 97, no. 7, pp. 2121-2129, 2001.
[3] C. Voermans, W. P. M. van Heese, I. de Jong, W. R. Gerritsen, and C. E. van der Schoot, "Migratory behavior of leukemic cells from acute myeloid leukemia patients," Leukemia, vol. 16, no. 4, pp. 650-657, 2002.

[4] J. A. Burger, A. Spoo, A. Dwenger, M. Burger, and D. Behringer, "CXCR4 chemokine receptors (CD184) and $\alpha 4 \beta 1$ integrins mediate spontaneous migration of human $\mathrm{CD} 34^{+}$ progenitors and acute myeloid leukaemia cells beneath marrow stromal cells (pseudoemperipolesis)," British Journal of Haematology, vol. 122, no. 4, pp. 579-589, 2003.

[5] S. Tavor, I. Petit, S. Porozov et al., "CXCR4 regulates migration and development of human acute myelogenous leukemia stem cells in transplanted NOD/SCID mice," Cancer Research, vol. 64, no. 8, pp. 2817-2824, 2004.

[6] A. Avigdor, P. Goichberg, S. Shivtiel et al., "CD44 and hyaluronic acid cooperate with SDF-1 in the trafficking of human CD $34^{+}$stem/progenitor cells to bone marrow," Blood, vol. 103, no. 8, pp. 2981-2989, 2004.

[7] L. Jin, K. J. Hope, Q. Zhai, F. Smadja-Joffe, and J. E. Dick, "Targeting of CD44 eradicates human acute myeloid leukemic stem cells," Nature Medicine, vol. 12, no. 10, pp. 1167-1174, 2006.

[8] R. Quéré, S. Andradottir, A. C. M. Brun et al., "High levels of the adhesion molecule CD44 on leukemic cells generate acute myeloid leukemia relapse after withdrawal of the initial transforming event," Leukemia, vol. 25, pp. 515-526, 2010.

[9] A. Colmone, M. Amorim, A. L. Pontier, S. Wang, E. Jablonski, and D. A. Sipkins, "Leukemic cells create bone marrow niches that disrupt the behavior of normal hematopoietic progenitor cells," Science, vol. 322, no. 5909, pp. 1861-1865, 2008.

[10] F. Ishikawa, S. Yoshida, Y. Saito et al., "Chemotherapyresistant human AML stem cells home to and engraft within the bone-marrow endosteal region," Nature Biotechnology, vol. 25, no. 11, pp. 1315-1321, 2007.

[11] S. L. Ellis, J. Grassinger, A. Jones et al., "The relationship between bone, hemopoietic stem cells, and vasculature," Blood, vol. 118, no. 6, pp. 1516-1524, 2011. 
[12] M. B. Meads, R. A. Gatenby, and W. S. Dalton, "Environmentmediated drug resistance: a major contributor to minimal residual disease," Nature Reviews Cancer, vol. 9, no. 9, pp. 665-674, 2009.

[13] S. M. Garrido, F. R. Appelbaum, C. L. Willman, and D. E. Banker, "Acute myeloid leukemia cells are protected from spontaneous and drug-induced apoptosis by direct contact with a human bone marrow stromal cell line (HS-5)," Experimental Hematology, vol. 29, no. 4, pp. 448-457, 2001.

[14] L. A. Hazlehurst, N. Valkov, L. Wisner et al., "Reduction in drug-induced DNA double-strand breaks associated with $\beta 1$ integrin-mediated adhesion correlates with drug resistance in U937 cells," Blood, vol. 98, no. 6, pp. 1897-1903, 2001.

[15] T. Matsunaga, N. Takemoto, T. Sato et al., "Interaction between leukemic-cell VLA-4 and stromal fibronectin is a decisive factor for minimal residual disease of acute myelogenous leukemia," Nature Medicine, vol. 9, no. 9, pp. 1158-1165, 2003.

[16] P. S. Becker, K. J. Kopecky, A. N. Wilks et al., "Very late antigen-4 function of myeloblasts correlates with improved overall survival for patients with acute myeloid leukemia," Blood, vol. 113, no. 4, pp. 866-874, 2009.

[17] T. Matsunaga, F. Fukai, S. Miura et al., "Combination therapy of an anticancer drug with the FNIII14 peptide of fibronectin effectively overcomes cell adhesion-mediated drug resistance of acute myelogenous leukemia," Leukemia, vol. 22, no. 2, pp. 353-360, 2008.

[18] P. S. Becker, F. R. Appelbaum, S. Chien et al., "Oral small molecule inhibitor of VLA-4 overcomes adhesion mediated chemotherapy resistance of acute myeloid leukemia (AML) blasts in vitro, without impairment of normal blood cell recovery when combined with chemotherapy in vivo," Blood, vol. 112, p. 858, 2008.

[19] Z. Zeng, I. J. Samudio, M. Munsell et al., "Inhibition of CXCR4 with the novel RCP168 peptide overcomes stroma-mediated chemoresistance in chronic and acute leukemias," Molecular Cancer Therapeutics, vol. 5, no. 12, pp. 3113-3121, 2006.

[20] K. Beider, M. Begin, M. Abraham et al., "CXCR4 antagonist 4F-benzoyl-TN14003 inhibits leukemia and multiple myeloma tumor growth," Experimental Hematology, vol. 39, no. 3, pp. 282-292, 2011.

[21] A. Mandawat, W. Fiskus, K. M. Buckley et al., "Pan-histone deacetylase inhibitor panobinostat depletes CXCR4 levels and signaling and exerts synergistic antimyeloid activity in combination with CXCR4 antagonists," Blood, vol. 116, no. 24, pp. 5306-5315, 2010.

[22] Z. Zeng, Y. X. Shi, I. J. Samudio et al., "Targeting the leukemia microenvironment by CXCR4 inhibition overcomes resistance to kinase inhibitors and chemotherapy in AML," Blood, vol. 113, no. 24, pp. 6215-6224, 2009.

[23] B. Nervi, P. Ramirez, M. P. Rettig et al., "Chemosensitization of acute myeloid leukemia (AML) following mobilization by the CXCR4 antagonist AMD3100," Blood, vol. 113, no. 24, pp. 6206-6214, 2009.

[24] F. De Toni, C. Racaud-Sultan, G. Chicanne et al., "A crosstalk between the Wnt and the adhesion-dependent signaling pathways governs the chemosensitivity of acute myeloid leukemia," Oncogene, vol. 25, no. 22, pp. 3113-3122, 2006.

[25] L. A. Hazlehurst, R. F. Argilagos, and W. S. Dalton, " $\beta 1$ integrin mediated adhesion increases Bim protein degradation and contributes to drug resistance in leukaemia cells," British Journal of Haematology, vol. 136, no. 2, pp. 269-275, 2007.
[26] M. Konopleva, S. Konoplev, W. Hu, A. Y. Zaritskey, B. V. Afanasiev, and M. Andreeff, "Stromal cells prevent apoptosis of AML cells by up-regulation of anti-apoptotic proteins," Leukemia, vol. 16, no. 9, pp. 1713-1724, 2002.

[27] Y. Tabe, L. Jin, Y. Tsutsumi-Ishii et al., "Activation of integrinlinked kinase is a critical prosurvival pathway induced in leukemic cells by bone marrow-derived stromal cells," Cancer Research, vol. 67, no. 2, pp. 684-694, 2007.

[28] E. J. C. Rombouts, B. Pavic, B. Lowenberg, and R. E. Ploemacher, "Relation between CXCR-4 expression, Flt3 mutations, and unfavorable prognosis of adult acute myeloid leukemia," Blood, vol. 104, no. 2, pp. 550-557, 2004.

[29] S. Konoplev, G. Z. Rassidakis, E. Estey et al., "Overexpression of CXCR4 predicts adverse overall and event-free survival in patients with unmutated FLT3 acute myeloid leukemia with normal karyotype," Cancer, vol. 109, no. 6, pp. 1152-1156, 2007.

[30] A. C. Spoo, M. Lübbert, W. G. Wierda, and J. A. Burger, "CXCR4 is a prognostic marker in acute myelogenous leukemia," Blood, vol. 109, no. 2, pp. 786-791, 2007.

[31] E. Tavernier-Tardy, J. Cornillon, L. Campos et al., "Prognostic value of CXCR4 and FAK expression in acute myelogenous leukemia," Leukemia Research, vol. 33, no. 6, pp. 764-768, 2009.

[32] A. Kalinkovich, S. Tavor, A. Avigdor et al., "Functional CXCR4-expressing microparticles and SDF-1 correlate with circulating acute myelogenous leukemia cells," Cancer Research, vol. 66, no. 22, pp. 11013-11020, 2006.

[33] R. B. Walter, T. A. Alonzo, R. B. Gerbing et al., "High expression of the very late antigen- 4 integrin independently predicts reduced risk of relapse and improved outcome in pediatric acute myeloid leukemia: a report from the children's oncology group," Journal of Clinical Oncology, vol. 28, no. 17, pp. 2831-2838, 2010.

[34] T. Südhoff, A. Wehmeier, K. O. Kliche et al., "Levels of circulating endothelial adhesion molecules (sE-selectin and sVCAM-1) in adult patients with acute leukemia," Leukemia, vol. 10, no. 4, pp. 682-686, 1996. 


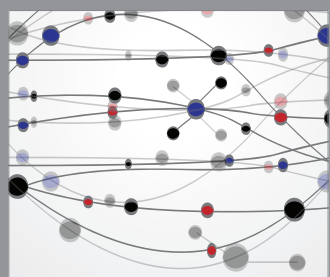

The Scientific World Journal
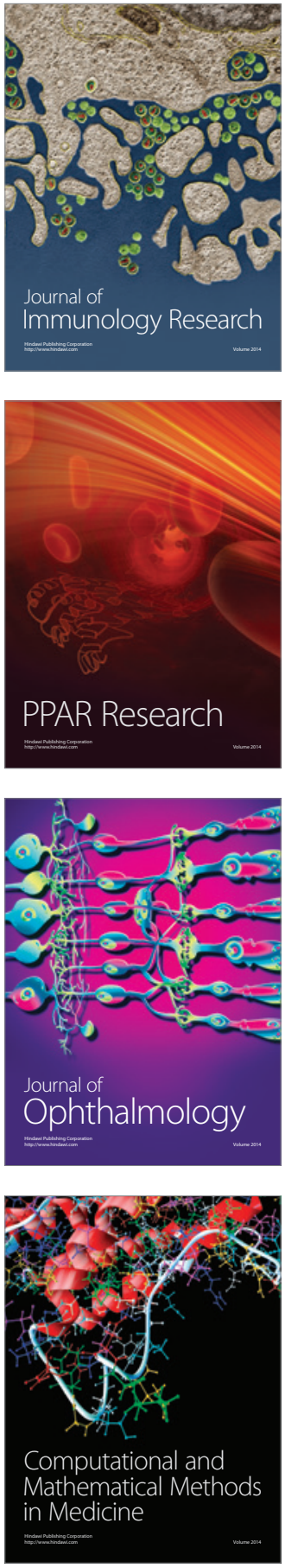

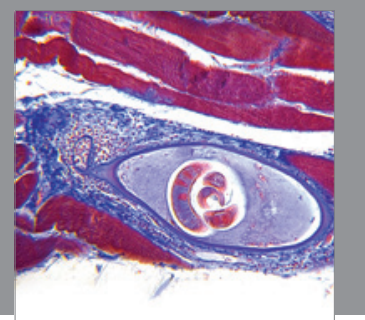

Gastroenterology

Research and Practice
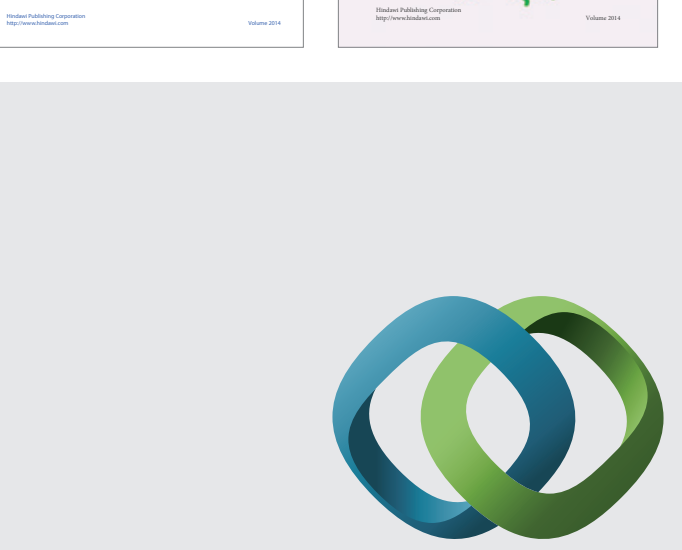

\section{Hindawi}

Submit your manuscripts at

http://www.hindawi.com
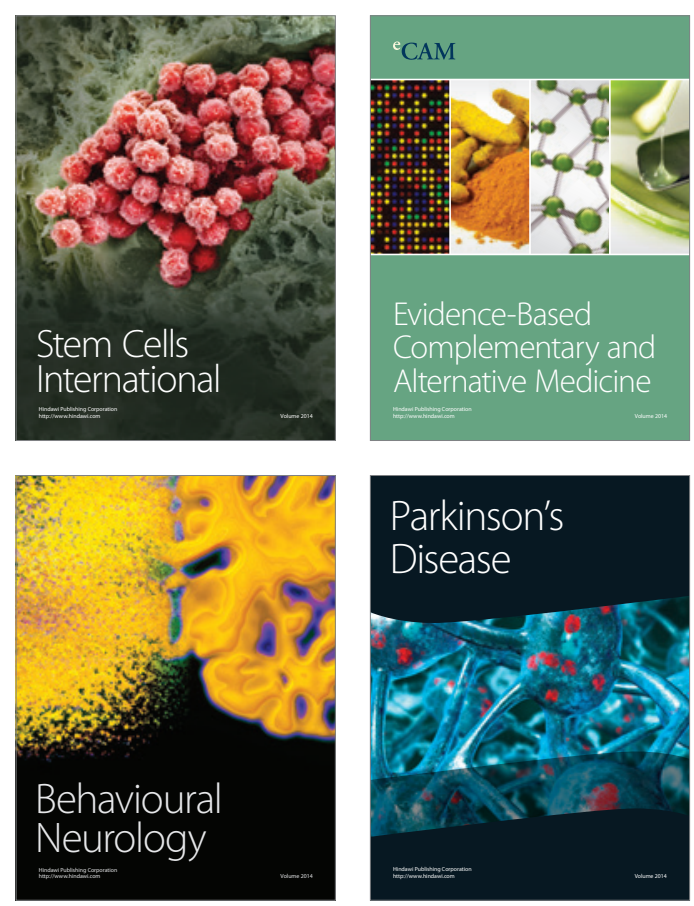

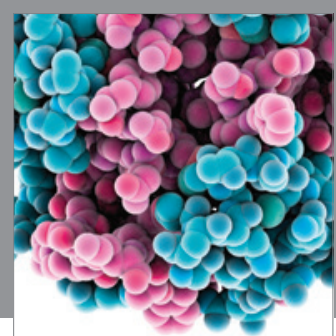

Journal of
Diabetes Research



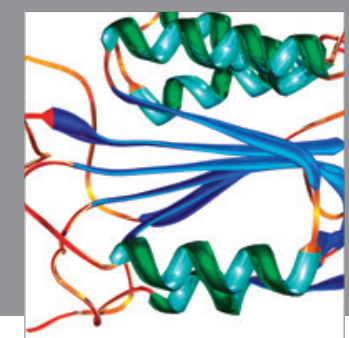

Disease Markers
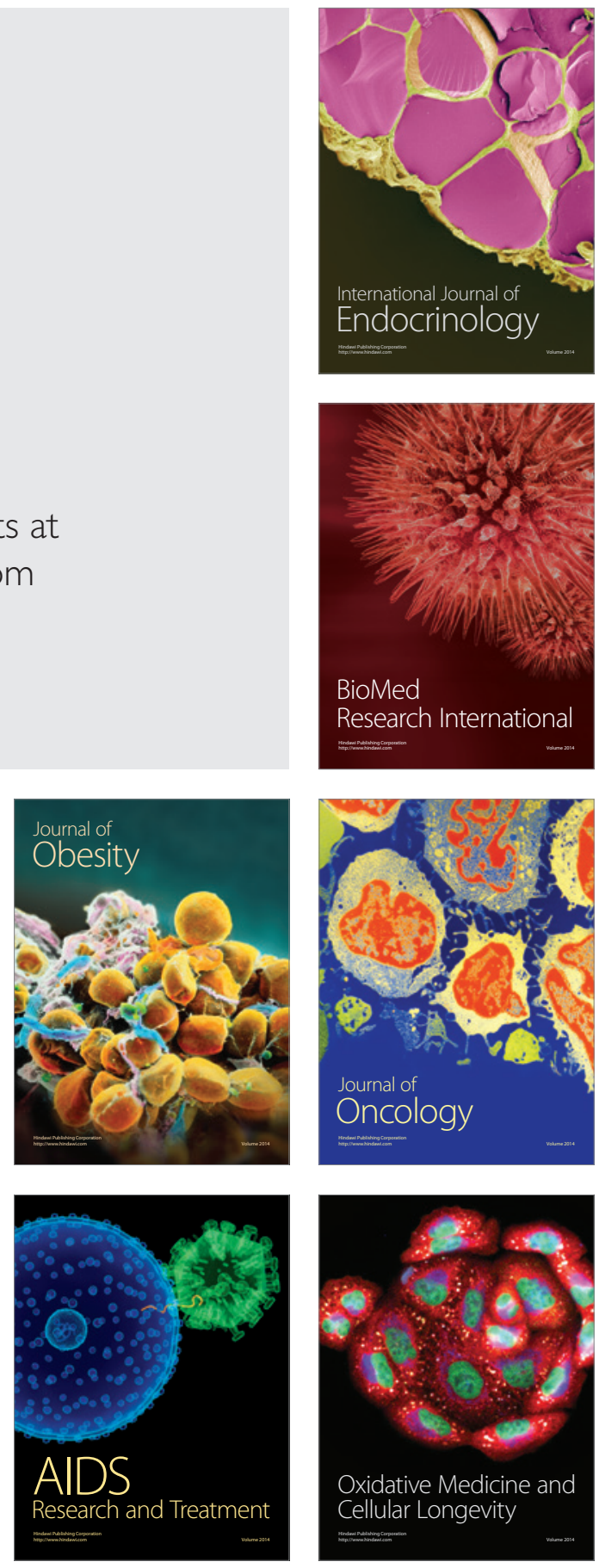